\title{
Empirical Effects of Online Retailing Recommendations
}

\author{
Hsiaoping Yeh \\ National Kaohsiung University of Science \& Technology, Kaohsiung City, Taiwan \\ Fenghung Kuo \\ College of Management, Kaohsiung City, Taiwan
}

\begin{abstract}
Online recommendation solves the current information overload problem in the online retailing businesses. Given relevant products by adopting recommendation algorithms, online shoppers can save time on searching and browsing for contents that they are interested in. Hence, in the increasing interests of online retailers, an empirical study was conducted to light the effectiveness of different entitled recommendations reflect on online shoppers. Working with a simulated online shopping establishment, the findings provide online retailers important guidelines regarding online customers' behaviors.
\end{abstract}

Keywords: recommender system, online recommendation, online shopping, customer behavior

\section{Introduction}

Recommender systems, designed to offer products of interest to customers, use people's opinions about items in an information domain to help people choose other items using the item taxonomy, attributes, and descriptions (Srikumar, 2004). Based on Business 2.0 Magazine, up to $30 \%$ of an online retailer's sales may be driven by product suggestions. Rosen and Olshavsky (1987) found that recommendations can speed up the people choices. East, Lomax, and Narain's (2001) study indicated that over 30\% of customers switch their choice due to recommendations. Online shopping recommendations are the Internet's answers to the old-fashioned up- and cross- sales, such as "you will probably like" and "people who bought this also bought". According to Forrest Research analyses, online recommender systems can account for $10 \%$ to $30 \%$ of online retailer's sales. Moreover, from the results of a survey conducted by Burke (2002), over $80 \%$ of customers were enthusiastic about using the Internet to search for product information and to compare and evaluate alternatives; yet a significant percentage (21\%) of online shoppers has negative reactions to such recommendation services. The eBay bought Shopping.com, an online comparison shopping site that provides recommendation services for $\$ 620$ million, commended by The Economist on $2005 / 6 / 4$, stressing the importance of recommender systems on e-commerce.

Since, recommender systems become important decision aids in the e-marketplace and an integral part of the business models of many firms (Adomavicius, Bockstedt, Curley, \& Zhang, 2013), most recommender systems use customer experienced items as inputs for the system's computational techniques (based on methodologies, they form statistics, data mining, or machine learning). Yet, recommender systems, serving

\footnotetext{
Hsiaoping Yeh, Ph.D., Associate Professor, Department of Marketing and Distribution Management, National Kaohsiung University of Science \& Technology, Kaohsiung City, Taiwan.

Fenghung Kuo, M.S., Ph.D. Program in Management, College of Management, Kaohsiung City, Taiwan.
} 
only recommendations, estimate items that have not been purchased by customers who will like the items. Therefore, there must be a research space on recommender systems that have actual effects and effectiveness on online shopping sites and online shoppers.

Recommender systems designers and researchers have primarily focused on delivering accurate recommendations with the development and improvement of the algorithms. Although many of the well-tuned algorithms, with a wide range of algorithmic approaches to problem accuracy have been solved, delivering these accurate predictions to users in a way that creates the best retailing purposes in terms of strengthening customer satisfaction/loyalty, increasing sales, enhancing purchase intention experience for the online retail practices remains an open problem. The effects of presentations are much less studied. The online customer behavioral implications of using recommender systems in the online shopping are likely to be the next area where significant improvements can be made. Very little research has explored how the cues provided by recommender systems influence online consumer behaviors. Therefore, the purpose of this study is to investigate the impacts of online recommender systems, particularly the effectiveness of different entitled recommenders on online shoppers.

\section{Literature Review and Study Hypotheses}

The psychological literature on conformity suggests that in the course of helping people make choices, recommender systems probably affect users' opinions of the items (Ariely, Lewenstein, \& Prelec, 2003; Epley \& Gilovich; 2010). Researchers in behavioral decision-making and applied psychology have found that people are often influenced by elements in the environment and their preferences are constructed (Chapman \& Johnson, 2002, Lichtenstein \& Slovic, 2006). In e-commerce, where affinity marketing is playing an increasingly important role, the purpose is to intensify visibility, motivate traffic, and boost sales (Bloom, Hoeffler, Keller, \& Basurto Meza, 2006). Given an online recommendation solution, according to which a recommendation product is selected based on customers behavior records in each phase of the online purchase decision process, the recommendation ones with richer content can be more effectively provided to the customers and the conversion rate of the online decision is hopefully improved.

The determination of customers' making the final decision on online purchase involves the comparison of alternatives for mediating the perceived need-state of the customers (Volk, 2001). Therefore, recommender systems, characterized as one style of customer decision support system (DSS) (Xiao \& Benbasat, 2007), are nowadays a clearly an important influence on customers' purchasing decisions (Zahir, 2002) since it solves the current information overload problem in the online world, improves decision quality, and increases confidence in product choice (Häubl \& Trifts, 2000; B. K. Lee \& W. N. Lee, 2004; Hostler, Yoon, \& Guimaraes, 2005). By clicking on recommendation items, online shoppers often alter their decision rapidly (Butler \& Peppard, 1998). As studies have shown, if customers surfing a Website see a hyperlink that they view as relevant, they may take immediate action, such as clicking and then making a purchase if it is linked to a product with the price and features of all alternatives (Celsi \& Olson, 1988; East et al., 2001). While traditional media face problems related to clutters and segments, the online recommender systems provide an opportunity to stand out and deliver messages that employ behavioral targeting to enhance the relevance of the message to customers (Pagnani, 2004; Nasco \& Bruner, 2008). Since the causes leading to changes in customer preferences and demands are recommenders, purchase advocacy, or referral systems (East et al., 2001), in the various steps of customer decision-making processes in e-commerce environments, recommendation techniques have been 
widely used as criteria for searching products on the Internet and generating advice and recommendations for online customers (Grenci \& Todd, 2002). Given the potential advantages of recommenders, it is important for researchers to explore the contexts in which customers are attentive to recommendations. This refers to the ability to deliver the message and gain customers' attention as well as for e-retailers to increase sales.

With the growing number of consumers who find values in recommender systems, marketers have little choice but no incorporate them into their online stores (Zahir, 2002; Ochi, Rao, Takayama, \& Nass, 2010). Past research has explored recommender systems based on software generated algorithms that aggregates different types of information provided by a company, an independent party, or customers (Senecal \& Nantel, 2004; Aggarwal \& Vaidyanathan, 2005; Ochi et al., 2010). While the use of recommendations is growing quickly, specific characteristics that customers want from these services have not yet to be thoroughly researched (Aljukhadar \& Senecal, 2011). It is especially important in the context of recommendations that they must efficiently and effectively aid customer decision-making rather than overwhelm them with additional and irrelevant information (Häubl \& Murray, 2006). By designing relevant and easy to use recommendations that include only targeted features that customers want, marketers may be able to improve customer satisfaction and generate more sales. Further, trustworthy and credible recommendations (but not fully directly to customers' action in terms of placing orders) by researchers suggest the sources of such systems come from independent sources rather than from retailers (West et al., 1999; Weathers, Sharma, \& Wood, 2007; Lepkowska-White, 2013). Therefore, focusing the recommendation based on a variety of information coming from the host retailer, the effects of product types on customers' responses to such recommendation on the actual purchases, is examined in this study. With customers' responses to recommendations coming directly from the host retailers, e-retailers can personalize a relationship with their customers.

Recommender systems adopt algorithms in order to offer users alternatives of their target products in many domains. A good or better recommender system is not able to assist users to look for a single item that closely matches their queries but also provides all items that are likely to fit the users' interests (McSherry, 2003). McSherry (2003) argued that current recommender systems take too much effort to find similar products to the users' target query, based on either content-based or collaborative filtering principles, and this can result in recommendation failure. In online retailing, in order to promote sales, companies often separate different anchors to describe recommendations titled with such phrases as "you are probably interested (or "featured recommendation"; "relative items you've viewed")", "the best buy" (or "the hit items"), "others also viewed", and "the most viewed", on a certain product pages where the shoppers are viewing. Furthermore, Aggarwal and Vaidyanathan (2005) found that customers prefer recommendations that match their preferences with product features rather than recommended items generated based on a variety of other sources. However, Ochi et al. (2010) derived the opposite result, finding that customers prefer the latter over the former. Hence, while prior research on recommender systems has focused mostly on developing and evaluating different algorithms that generate recommendations, this study instead identifies other important aspects of recommender systems, namely, recommender systems related to product, online shoppers' responding behaviors, and consequences influenced by the recommendation products with different recommendation titles. That is, this study focuses on online retail consumer reactions related to the acquisition and buying intention of products presented on recommenders, and separates the outcomes of decision-making from the processes of decision-making.

Therefore, abstracting from all of the literature, this study posits that different entitled recommenders have different impacts on online shoppers' responses while they are viewing the target products as well as different 
contributions to their shopping intentions, altering their purchase behaviors. The effectives of recommendations in this study are implicitly posted from e-retailers. More specifically, this study observes the effectiveness of certain recommender systems:

H1: The duration time of viewing a target product page with recommenders is significantly shorter than that without recommenders.

$\mathrm{H} 2$ : Among product types, the duration time of viewing recommendation product page is significantly different.

H3: Among entitled recommenders, there are significantly different impacts on the tendency to click the recommendation products.

H4: Among entitled recommenders, there are significantly different impacts on the duration time of viewing recommendation product pages.

H5: Among entitled recommenders, there are significantly different impacts on online shoppers' adding recommendation products to the tracking lists.

H6: Among entitled recommenders, there are significantly different impacts on online shoppers' putting recommendation products in their shopping carts.

H7: Among entitled recommenders, there are significantly different impacts on online shoppers' replacing the target products in shopping carts with the recommendation products.

H8: Among entitled recommenders, there are significantly different impacts on online shoppers' recommendations to others.

\section{Methodology}

The purpose of this study was to investigate the impacts of recommendation products named differently with "you are probably interested", "the best buy", "others also viewed", and "the most viewed" in terms of how long the recommendation product is clicked after the target is browsed, which product types make the recommendation effective, what following actions on the recommendation products, such as adding to tracking list, putting in shopping carts and replacing the target products in shopping carts, and sociality effect on the different entitled recommendation products. The measurement of the effectiveness of a recommendation is the page viewing time and the actions taken by online shoppers when viewing recommendation products.

To investigate the e-commerce effects on customer behaviors, full ranges of scenarios designed for testifying and collecting customer responses, rather than the reality recorded in the real online retailing sites, are believed to make full comprehension of e-commerce customer behaviors in the design and management of marketing plans. Hence, the data collection instrument in this study is a specially designed online shopping site, resembling the interfaces of one of the top three biggest online retail sites in Taiwan. Since the simulated online retail program is not able to let study subjects search products by keywords at free will, products are all aligned by product categories on the main page. Generally, in the top three biggest Taiwan online shopping sites, goods types can be classified into nine major categories: foods, sports \& leisure, 3C, outfits, culture \& education, cosmetics \& health care, commodities, office supplies, and home appliances. For each product category, 10 items with pictures, in different sub-categories, are shown on the main page, scrolling through the list in a category with arrow keys. All products shown in the main page are noted as target products in this study.

When a target product page is clicked and opened, showing its detailed description, there are six recommendation products on each of four rows of the recommendation products entitled as "you are probably 
interested", "the best buy", "others also viewed", and "the most viewed", shown on the bottom of the target product page. The reason for listing six recommendation products as being reasonable and acceptable compared with other numbers of recommendation sizes for the targets is based on the finding by McSherry (2003). The selection and assigning criteria of the recommendation products with the numbers of descriptive features/attributes for each target item is based on McSherry's compromise-driven retrieval algorithm (McSherry, 2003), which not only recommends highly similar goods to users but also take consideration of the users' possible compromise attitude, being proven as good or better in some objective sense and considerably acceptable for recommender systems (Burkhard, 1998; McSherry, 2003).

In order to testify that the hypotheses that the titles of recommender system are effective to customer online behaviors, the six products on each row of titled recommendation are purposely picked, but randomly allocated on each row, to be one-to-one similar products with the same features and attributes across four titles, yet not necessarily with same product name and manufacturers/brands. That is, recommendation products appearing on each entitled row are conditionally similar.

Note that only recommendations are shown on the target product page. That is, there are no further any recommendations on the recommendation product pages. The study subjects are all tracked by page clicks, the duration time of product page viewing, and the actions on the viewing product (i.e., adding to the tracking list, putting in shopping cart, and forwarding to others) on the simulated shopping site.

In order to keep track of the page viewing time of all items correctly, the target product page will be closed once a recommendation product page is clicked and the opened product page, either the target or recommended one, will be closed once another target product page is clicked. Once a product, either target or recommended, has been taken into action by a study subject (e.g., adding it to the tracking list), the page is closed.

The simulated shopping themes are designed twofold: with and without recommender system. Study participants are randomly assigned to either with or without shopping theme, but the demographics of genders and ages are controlled to be almost evenly distributed.

The study subjects were students from both college and graduate school, who asked for voluntarily to participate. Before the experiment, each subject was instructed to use the simulated shopping site and told that all layouts resemble the Web shopping center. To avoid data distortion, subjects were asked to do their best not to click the same product pages twice. The completion data were also checked again.

The collection time for each subject on the shopping theme with recommender system was set at one hour or when subjects did not want to continue the procedure. However, the subjects on the other were only for 30 minutes. Each dataset was recorded with data fields of: (1) subject's assigned sequential number; (2) the computer system time; (3) subject's age; (4) subject's gender; (5) the viewing product page is the target or recommendation one; (6) one of the four recommender titles under the viewing recommendation product; (7) viewing time (in seconds) of the product page; (8) the product type (using product category not product sub-code in this study); (9) if the product is added to the tracking list; (10) if the product is put into shopping cart; (11) if it is a replacement of target item with recommendation one; and (12) if the product is recommended to others by the subject.

There were 278 subjects participating in the study, evenly divided on with and without recommender systems. The number of datasets from the shopping theme without recommender system is 2,813 and that collected from the theme with recommendation is 4,894 . Among the subjects for the shopping theme with 
recommender system, there are eight females who did not click any recommendation product pages and 36 males that also did not.

\section{Results}

The demography of subjects and datasets are shown in Table 1 .

Table 1

Demography of Subjects and Datasets

\begin{tabular}{llllll}
\hline & & \multicolumn{2}{c}{ For subjects (out of 278) } & \multicolumn{2}{c}{ For datasets } \\
\cline { 3 - 6 } & & Without (\%) & With (\%) & Without (out of 2,813, \%) & With (out of 4,894, \%) \\
\hline \multirow{2}{*}{ Gender } & Female & 30 & 29 & 45 & 78 \\
& Male & 21 & 20 & 55 & 22 \\
& 18-22 years old & 20 & 22 & 31 & 32 \\
\multirow{4}{*}{ Age } & $23-29$ years old & 16 & 16 & 30 & 31 \\
& $30-35$ years old & 7 & 7 & 32 & 21 \\
& $36-40$ years old & 5 & 5 & 6 & 12 \\
\hline
\end{tabular}

A $t$-test, for H1, with non-equal variance, on data of shopping themes between with and without recommender systems is shown in Table 2. The data used for H2-H8, with ANOVA shown in Table 3, are those in the shopping theme with recommender systems.

Table 2

The t-tests for Hypothesis H1

\begin{tabular}{lllllll}
\hline Data & Shopping theme & Mean & Std. & $N$ & $t$ & Sig. (2-tailed) \\
\hline \multirow{2}{*}{ Viewing time } & Without & 62.73 & 17.13 & 2,813 & \multirow{2}{*}{27.176} & 0.000 \\
& With & 47.56 & 31.85 & 4,894 & & \\
\hline
\end{tabular}

Table 3

ANOVA for the Hypotheses H2-H8

\begin{tabular}{|c|c|c|c|c|c|c|c|}
\hline Hypothesis & Source & Data & SS & $d f$ & MS & $F$ & Sig. \\
\hline \multirow{2}{*}{$\mathrm{H} 2$} & Product type & \multirow{2}{*}{$\begin{array}{l}\text { Viewing time of } \\
\text { recommendations }\end{array}$} & 1353.543 & 8 & 169.193 & \multirow{2}{*}{12.926} & \multirow{2}{*}{0.000} \\
\hline & Error & & 29686.056 & 2268 & 13.089 & & \\
\hline \multirow{2}{*}{$\mathrm{H} 3$} & Title & \multirow{2}{*}{$\begin{array}{l}\text { Frequency of targets } \rightarrow \\
\text { recommendations }\end{array}$} & 0.371 & 3 & 0.124 & \multirow{2}{*}{244.967} & \multirow{2}{*}{0.000} \\
\hline & Error & & 0.209 & 552 & 0.000 & & \\
\hline \multirow{2}{*}{$\mathrm{H} 4$} & Title & \multirow{2}{*}{$\begin{array}{l}\text { Viewing time of } \\
\text { recommendations }\end{array}$} & 24601.782 & 3 & 8200.594 & \multirow{2}{*}{633.380} & \multirow{2}{*}{0.000} \\
\hline & Error & & 29429.301 & 2273 & 12.947 & & \\
\hline \multirow{2}{*}{ H5 } & Title & \multirow{2}{*}{ Tracking list } & 0.124 & 3 & 0.041 & \multirow{2}{*}{64.623} & \multirow{2}{*}{0.000} \\
\hline & Error & & 0.197 & 308 & 0.001 & & \\
\hline \multirow{2}{*}{ H6 } & Title & \multirow{2}{*}{ Shopping cart } & 0.060 & 3 & 0.020 & \multirow{2}{*}{28.571} & \multirow{2}{*}{0.000} \\
\hline & Error & & 0.128 & 240 & 0.001 & & \\
\hline \multirow{2}{*}{$\mathrm{H} 7$} & Title & \multirow{2}{*}{ Replacement } & 0.022 & 3 & 0.007 & \multirow{2}{*}{16.596} & \multirow{2}{*}{0.000} \\
\hline & Error & & 0.076 & 172 & 0.000 & & \\
\hline \multirow{2}{*}{ H8 } & Title & \multirow{2}{*}{ Sociality } & 0.029 & 3 & 0.010 & \multirow{2}{*}{16.111} & \multirow{2}{*}{0.000} \\
\hline & Error & & 0.072 & 120 & 0.001 & & \\
\hline
\end{tabular}

Recommender systems appearing on the subjects' viewing target product pages are significantly effective with the evidence of that their target product page viewing time becomes shorter while the pages are with 
recommender systems appearing with their featured recommendations. Note that the standard deviation of the target product page viewing time for the shopping theme with recommender systems is large, implying that subjects' reactions to the recommender systems were varied. The findings on the further hypotheses reveal some clues.

Indeed, there are significant differences in the subjects' viewing recommendation product pages among product types. Based on the results of the post hoc test (with Sheffe), the time of viewing recommendation items among product categories is that on $3 \mathrm{Cs}$ and home appliances is the longest; followed by on outfits; on sports \& leisure and cosmetics \& health care; and then on foods, culture \& education, and commodities. On office supplies it is the shortest. The time among entitled recommenders (i.e., testing H4) also shows significant differences on viewing the recommendation items. The time length order among four titles is, from the shortest to the longest, "the best buy", "the most viewed", "others also viewed", and "you are probably interested".

Testing H3 is comparing the frequencies of four entitled recommenders for an individual subject who clicked the first recommendation products after the target item. The result shows there is a significant impact among different entitled recommender systems. With Sheffe post hoc test, subjects' clicking the first recommendation items after they viewed target products indicates that subjects' attention was most drawn by the recommendation items within the "the best buy" recommendation, and the least by the ones within "you are probably interested"; "the most viewed", and "others also viewed" that are in between.

H5-H8 compare the ratios of taking actions (including adding items to the tracking list, putting items in shopping cart, making replacement of target items with recommendations, and forwarding recommending items to folks via emails) among all four titles of recommenders for each subject, but the subjects who did not take any one of actions are screened out. There were 78 subjects who have added at least one of the recommendation items to their tracking lists, 61 subjects put recommendation ones in shopping carts, 44 ones replaced target items with recommendations, and 31 ones forwarded recommendation product information to others.

Given the subjects who have taken any actions on recommendation products, with ANOVA and Sheffe post hoc tests, for all actions expressed in this study, there is strong evidence that, on average, "the best buy" recommendation products are most acceptable by the subjects and followed by the ones within "the most viewed", "others also viewed", and "you are probably interested", which is the same as the previous hypothesis testing results.

Summarizing from all the findings, online shoppers' attention on their target interests would be distracted by recommender systems. When online shoppers purchase products with low customer involvement, such as 3C and home appliances, they tend to consult with recommendations; yet they intend not to do so for the products with high customer involvement, such as foods, culture \& education, commodities, and office supplies. The wordings of recommender systems are one important key to acceptance of recommenders for online shoppers. "The best buy" recommender implies the "product assurance" by others and therefore its acceptance and effectiveness are apparently higher than other entitled recommenders.

\section{Conclusion Remarks and Implications}

This study contributes to addressing recommender system for e-commerce application. Discarding the real click stream data for a case study, this study was especially designed for online shopping scenarios with different recommending settings. For research purposes, it investigates online customers' decision-making behaviors. 
Marketing managers make enormous efforts to sell their products and services to their customers. Their marketing activities are engaged in order to ensure the customers choose their products from a full range of competing products. The objective of this study is to discover how online shoppers react to recommender systems that provide recommendation items to shoppers' viewing their interested target products. With a purposely designed web retailing setting, the different effects of entitled recommender systems are testified. In more detail, the effectiveness of recommender system investigated in this study includes the acceptance and usefulness of its appearance, its acceptance and usefulness among different product types, its acceptance and usefulness as well as the practical/commercial values, in terms of adding recommendation items to a tracking list, putting them into shopping carts, replacing target items with recommendations, and endorsing them by passing the recommendations to others, among different recommender titles.

While several authors have questioned the assumption that the most similar case is always the one that is most acceptable or useful (Burkhard, 1998; Smyth\&Keane, 1998; McSherry, 2003), this study demonstrates that recommender systems with different advocacy wordings affect online customers' purchase intentions. The analytic results give web retailers confidence that recommender systems are, at least to some extent, influencing customers to seek out additional choices and chances to direct and promote their purchase intention. Secondly, recommender systems can be broadly classified based on the type of products for which they offer recommendations. These high involvement products are often purchased based on customers' past usages and experiences. Therefore, from the planned behavior perspective, the time spent on making such purchases is not long, particularly for the goods for family usage and by the mature and rational customers (Mathew, 2013; Tai, 2005). Hence, proven by this study, online customers would pay less much attention to the recommender systems. Srikumar (2004) suggested that recommender systems that focus on low involvement products make use of customers' personal preferences, purchase history, and click-stream data to offer recommendations. On the other hand, recommender systems for high involvement products typically take users' ephemeral needs and match them with a set of product attributes to offer recommendations.

The managerial implications made by this study are the adoption of the recommender systems on e-retailing Website. Lepkowska-White (2013) gave evidence that customers tend to hold more positive attitudes towards the source from third parties rather than that coming directly from the sellers. Yet, acceptance and usefulness of recommender systems provided directly from e-retailers might be gauged with online users' trust (Pu \& Chen, 2007; Wang \& Benbasat, 2007). This study first testifies the effectiveness of recommendation products appearing online, implying the providing sources being from e-retailers. However, the achievement of recommender systems, from only browsing to making further recommendation and even to showing purchase intention (i.e., adding to the tracking list and putting into shopping cart in this study) and to changing the made purchase decision (i.e., target product replacements in this study), indeed, reveals a certain degree of differentiation, based on different categories of product types and word phrasings used on recommenders. That is, this study suggests that e-retailers use and design the titles of recommender systems carefully.

The source of recommendation still counts. This study used high similar products but different titles on recommenders and the results show that online customers are inclined to trust in purchase approval from others. Hence, online shoppers not only pay attention to the information about products but also the sources of such recommendation. Affinity marketing focuses on finding customers in a certain product or topic, then offering that customer related products or information from other customers with similar interests. The central theme of affinity marketing is to utilize group affiliation (either customers or products) as a means of generating a strong 
and credible promotional program that is specifically tailored to the individual, but is within the context of the group. Both group and individual incentives are often an integral part of the affinity programs. In this study, personalized recommendation, implied with the usage of the recommender entitled "you are probably interested", requires online retailers a move to truly interactive Website. Furthermore, since in reality, the decision of recommender system for online retailers is influenced by suppliers or supplies due to prices or inventories, for instance, the product arrangement showing on product pages may not truly reflect on the products on Web pages in terms of recommendations entitled "the best buy", "the most viewed", etc. but fall into the category of merely retailers' promotion/sales.

This study only focuses on discovering the effects of recommender systems on e-retailing, particularly by leveraging different product types and different entitled recommendations, yet given all similar products. The effects of degrees of product similarities and incorporation of customers' online shopping behaviors and perceptions in an all-round manner (e.g., product knowledge, experience, and trust) would be another and the next research. Besides, recommendation products appeared in "other also bought" may not fit the featured similarity products with the target items but are for cross-sales purposes, which would also be our future study.

\section{References}

Adomavicius, G., Bockstedt, J. C., Curley S. P., \& Zhang, J. (2013). Do recommender systems manipulate consumer preferences? A study of anchoring effects. Information Systems Research, 24(4), 956-975.

Aggarwal, P., \& Vaidyanathan, R. (2005). Perceived effectiveness of recommendation agent routines: Search vs. experience goods. International Journal of Internet Marketing and Advertising, 2(1), 38-55.

Aljukhadar, M., \& Senecal, S. (2011). Usage and success factors of commercial recommendation agents: A consumer qualitative study of MyProductAdvisor.com. Journal of Research in Interactive Marketing, 5, 130-152.

Ariely, D., Lewenstein, G., \& Prelec, D. (2003). Coherent arbitrariness: Stable demand curves without stable preferences. Quarterly Journal of Economy, 118, 73-105.

Bloom, P. N., Hoeffler, S., Keller, K. L., \& Basurto Meza, C. E. (2006). How social-cause marketing affects consumer perceptions. MIT Sloan Management Review, 47(2), 49-55.

Burke, R. R. (2002). Technology and the customer interface: What consumers want in the physical and virtual store? Journal of the Academy of Marketing Science, 30(4), 411-432.

Burkhard, H. D. (1998). Extending some concepts of CBR-Foundations of case retrieval nets. In M. Lenz, B. Bartsch-Spörl, H. D. Burkhard, and S. Wess, (Eds.), Case-based reasoning technology (pp. 17-50). Berlin Heidelberg, New York: Springer-Verlag.

Butler, P., \& Peppard, J. (1998). Consumer purchasing ion the Internet: Processes and prospects. European Management Journal, 16(5), 600-610.

Celsi, R. L., \& Olson, J. C. (1988). The role of involvement in attention and comprehension processes. Journal of Consumer Research, 15(2), 210-214.

Chapman, G., \& Johnson, E. (2002). Incorporating the irrelevant: Anchorsin judgments of belief and value. In T. Gilovich, D. Griffin, and D. Kahneman (Eds.), Heuristics and biases: The psychology of intuitive judgment (pp. 120-138). Cambridge, UK: Cambridge University Press.

East, R., Lomax, W., \& Narain, R. (2001). Customer tenure, recommendation and switching. Journal of Consumer Satisfaction, Dissatisfaction and Complaining Behavior, 14, 46-54.

Epley, N., \& Gilovich, T. (2010). Anchoring unbound. Journal of Consumer Psychology, 20, 20-24.

Grenci, R. T., \& Todd, P. A. (2002). Solutions-driven marketing. Communications of the ACM, 45(3), 65-71.

Häubl, G., \& Murray, K. B. (2006). Double agents. MIT Sloan Management Review, 47(3), 8-12.

Häubl, G., \& Trifts, V. (2000). Consumer decision-making in online shopping environments: The effects of interactive decision aids. Marketing Science, 19(1), 4-21.

Hostler, R. E., Yoon, V. Y., \& Guimaraes, T. (2005). Assessing the impact of Internet agent on end users' performance. Decision Support Systems, 41(1), 313-323. 
Lee, B. K., \& Lee, W. N. (2004). The effect of information overload on consumer choice quality in an on-line environment. Psychology \& Marketing, 21(3), 159-183.

Lepkowska-White, E. (2013). Are they listening? Designing online recommendations for today's consumers. Journal of Research in Interactive Marketing, 7(3), 182-200.

Lichtenstein, S., \& Slovic, P. (2006). The construction of preference. Cambridge, UK: Cambridge University Press.

Mathew, S. S. (2013). Working women households and time-saving consumption. South Asia Research, 32, $215-231$.

McSherry, D. (2003). Similarity and compromise. The 5th International Conference on Case-Based Reasoning (ICCBR 2003). June 23-36, Trondheim, Norway.

Nasco, S., \& Bruner, G. (2008). Comparing consumer responses to advertising and non-advertising mobile communications. Psychology and Marketing, 25(8), 822-838.

Ochi, P., Rao, S., Takayama, L., \& Nass, C. (2010). Predictors of user perceptions of web recommender systems: How the basis for generating experience and search product recommendations affects user responses. International Journal of Human-Computer Studies, 68(8), 472-482.

Pagnani, M. (2004). Determinants of adoption of third generation mobile multimedia services. Journal of Interactive Marketing, 18(3), 46-59.

Pu, P., \& Chen, L. (2007). Trust-inspiring explanation interfaces for recommender systems. Knowledge-Based Systems, 20, 542-556.

Rosen, D. L., \& Olshavsky, R. W. (1987). A protocol analysis of brand choice strategies involving recommendations. Journal of Consumer Research, 14, 440-444.

Senecal, S., \& Nantel, J. (2004). The influence of online product recommendations on consumers' online choices. Journal of Retailing, 80(2), 159-169.

Smyth, B., \& Keane, M. (1998). Adaptation-guided retrieval: Questioning the similarity assumption in reasoning. Artificial Intelligence, 102, 249-293.

Srikumar, K. (2004). A framework of agent-based personalized recommender system for e-commerce. South Asian Journal of Management, 11(2), 66-80.

Tai, S. H. C. (2005). Shopping styles of working Chinese females. Journal of Retailing and Consumer Services, 12(3), $191-203$.

Volk, F. A. (2001). Internet users' attitudes and e-commerce behaviors (Ph.D. dissertation, Wichita State University).

Wang, W., \& Benbasat, I. (2007). Recommendation agents for electronic commerce: Effects of explanation facilities on trusting beliefs. Journal of Management Information Systems, 23(4), 217-246.

Weathers. D., Sharma, S., \& Wood, S. L. (2007). Effects of online communication practices on consumer perceptions of performance uncertainty for search and experience goods. Journal of Retailing, 83(4), 393-401.

West, P. M., Ariely, D., Bellman, S., Bradlow, E., Huber, J., Johnson, E., Kahn, ... Schkade, D. (1999). Agents to the rescue? Marketing Letters, 10(3), 285-300.

Xiao, B., \& Benbasat, I. (2007). E-commerce producer commendation agents: Use, characteristics, and impact. MIS Quarterly, 31(1), 138-209.

Zahir, S. (2002). Designing a knowledge-based interface for intelligent shopping agents. Journal of Computer Information Systems, 43(1), 31-41. 